\title{
Un hub archivistique en Afrique du Sud
}

\author{
Noor Nieftagodien et Matthieu Rey
}

Citer cet article : Nieftagodien Noor et Rey Matthieu (2021), " un hub archivistique en Afrique du Sud »,

Revue d'Histoire Contemporaine de l'Afrique, en ligne. URL :

https://oap.unige.ch/journals/rhca/article/view/stcnieftegodienrey

Mise en ligne : 8 octobre 2021

DOI : https://doi.org/10.51185/journals/rhca.2021.e565

\section{Résumé}

Un projet significatif doit donner naissance à un pôle (hub) résultant du rapprochement de plusieurs entités archivistiques à l'Université du Witwatersrand (Johannesbourg). Par sa visée, ce nouveau lieu deviendrait le premier centre d'archives indépendantes d'Afrique en Sud, en volume et en importance. Mais surtout, il est le fruit d'une intense réflexion sur la place des archives dans le projet démocratique qui anime le pays et dans les cursus universitaires. La place de ce hub archivistique ne peut se comprendre sans un regard sur I'Afrique du Sud depuis la fin de l'apartheid. La place de I'histoire et des archives y semble privilégiée au regard de ses voisins et de nombreux États, et elle dénote les espoirs et les soutiens qu'a suscité ce pays lors de la transition des années 1990. Un tel pôle permettrait d'opérer un rassemblement majeur de fonds d'archives au service d'un nouveau regard sur les centres indépendants de documentation et les pratiques qu'ils doivent générer.

Mots-clés : Johannesbourg ; Witwatersrand ; archives ; université 
Un projet significatif doit prochainement donner naissance à un pôle (hub) résultant du rapprochement de plusieurs entités archivistiques à l'Université du Witwatersrand (Johannesbourg, ci-après «Wits »). Par sa visée, ce nouveau lieu deviendrait le premier centre d'archives indépendantes d'Afrique en Sud, en volume et en importance. Mais surtout, il est le fruit d'une intense réflexion sur la place des archives dans le projet démocratique qui anime le pays et dans les cursus universitaires. Pour en comprendre l'importance, il faut dans un premier temps préciser les grandes catégories archivistiques, puis restituer comment ce hub résulte d'une histoire singulière des archives, pour enfin voir les enjeux épistémologiques et institutionnels qui sous-tendent les réflexions et travaux à l'origine de ce pôle archivistique. Cette création originale souligne comment archives et historiens interagissent mais aussi comment l'université dispose d'une mission particulière dans la conduite du recueil documentaire.

\section{Le paysage archivistique en Afrique du Sud}

L'Afrique du Sud présente une situation ambivalente: elle a connu historiquement un développement important des archives, mais de nombreux centres semblent ne plus attirer toute l'attention nécessaire à leur bon fonctionnement. Aussi, si les fonds sont exceptionnellement riches, au regard de nombreux autres États du Sud global, de par des traditions diverses de conservation, de valorisation et d'analyses critiques autour des ressources documentaires, leur avenir est incertain. Pour comprendre l'état actuel des fonds documentaires, il faut en tracer brièvement une typologie. Les archives peuvent être divisées en quatre grands ensembles dans ce pays: archives d'État, fonds privés, archives indépendantes et archives militantes.

Le premier grand ensemble relève des archives d'État qui se déclinent en trois niveaux géographiques - national, régional (couvrant une des neuf provinces du pays) et local. Toutes appartiennent largement à ce que l'on nomme des archives coloniales, en ce qu'elles ont été produites par l'État blanc d'apartheid, jusqu'en 1994. Elles sont essentielles pour connaître cette structure politique si particulière. Par les politiques développées pendant l'apartheid et le contrôle d'une rare ampleur et extrêmement tatillon de toutes les populations (système de pass, contrôle des habitats, déplacements, naissance, etc.), elles saisissent de nombreuses dimensions de la société sud-africaine. En cela, ces archives ne diffèrent pas des autres documentations d'État dans le monde, reflétant ses contours et sa trajectoire historique singulière.

Après 1994, les gouvernements successifs ont fait un effort de valorisation des fonds publics qui s'est traduit principalement par la construction de nouveaux bâtiments, par la multiplication des lieux de conservation, sans toujours penser à investir sur le long terme. Ce changement de politique reflète l'effervescence qui entoure l'Afrique du Sud des années de transition. Pour le nouvel État, il n'est pas possible en effet d'oublier les archives dans le processus de réforme qu'il entame pour tourner la page de l'apartheid. Dans cette perspective, des ressources importantes sont allouées afin de doter les nouvelles provinces de centres de conservations. Tout comme le démantèlement des bantoustans, donner aux publics sudafricains accès aux archives semble une priorité de l'État démocratique, ce qui passe par 
l'établissement de nouveaux dépôts, plus disséminés. Néanmoins, ce soutien ne s'avère pas pérenne. Aujourd'hui, une crise protéiforme affecte ces archives, avec des moyens moindres accordés à la conservation et à la mise en valeur. Cette situation suscite naturellement des inquiétudes sur l'avenir, renforçant l'importance des autres types d'archives.

À côté de cette première catégorie, viennent les archives privées, qu'elles soient celles de compagnies ou d'individus et dont le dénombrement dépasse les horizons du présent article. Cette catégorie désigne tous les lieux de conservation mis en place par une personne morale (compagnie, association) ou physique pour déposer et mettre en valeur ses documents. Aussi, de très nombreux lieux existent pour cela à travers le pays. Cette multiplication s'explique en partie par l'absence de sections spécifiques dans les archives d'État pour collecter des papiers privés et probablement par une certaine méfiance à l'égard de l'État central. Certaines personnalités et compagnies ont fait le choix de mettre en place leur propre fonds, ce qu'on désigne par archives privées.

À l'interstice entre ces deux grands ensembles, assez communs dans le monde, prennent place les archives indépendantes (independent archives) et les archives militantes (activist archives). Soulignons d'emblée que ces dénominations sont celles assumées par les acteurs. Ils souhaitent, dans le premier cas, insister sur le caractère non-étatique et, dans le second cas, sur la manière dont l'activité archivistique participe d'un combat politique plus large. Les premières sont le plus souvent entreposées dans les universités du pays. Comme institution autonome, l'université offre de jure, un cadre protégé, tout particulièrement à l'époque de l'apartheid : l'État n'avait - et n'a toujours - pas le droit d'intervenir sur le campus sans l'accord explicite de la direction de l'institution. Jalouses de leur statut, les universités se sont montrées très tôt enclines à être des espaces échappant aux officines sécuritaires d'État. En outre, le prestige de l'université comme centre de savoir en fait un lieu qui apparaît propice à une mise en valeur des fonds déposés, ou au moins un lieu garantissant des protections. C'est pourquoi, à partir des années 1970, lorsque le mouvement contre l'apartheid prend un essor inédit, les fonds d'archives que les universités conservent s'enrichissent rapidement, par des dépôts variés. Le fait de déposer des fonds archivistiques au sein d'une institution autonome de l'État confère aux archives leur caractère indépendant.

Le plus souvent, le centre d'archive au sein d'une université reflète la position de cette université, mais aussi les institutions, les personnes et organismes qui ont fait son histoire. II lui confère en quelque sorte son identité, même si celle-ci a pu changer au cours du temps. Par exemple, I'université de l'État libre (Bloemfontein) a très tôt accueilli les archives des personnalités du Parti National' ${ }^{1}$. Son identité afrikaner et son association au régime de l'apartheid jusque dans les années 1990 expliquent le choix opéré par ses dirigeants d'y déposer leurs papiers. À côté de cela, la Killie Campbel/ Library² a pu recevoir quantité de fonds privés : elle est en relation étroite avec l'Université du Kwazulu-Natal, ne serait-ce qu'en tant que dépôt des fonds concernant I'histoire de la province ${ }^{3}$. D'autres centres, comme Historical

\footnotetext{
1 URL : https://www.ufs.ac.za/library/libraries-divisions/archive-for-contemporary-affairs-home/collections/political-collection (consulté le 5 octobre 2021).

2 URL : https://campbell.ukzn.ac.za/?q=node/42 (consulté le 5 octobre 2021).

3 Depuis les archives de James Stuart (fonctionnaire colonial qui débute une grande enquête par entretiens oraux sur les origines des royaumes zoulous), en passant par la collection des dissertations des élèves reprenant les histoires des clans.
} 
Papers', à I'Université du Witwatersrand, se sont structurés autour de l'Anglican Church, de I'Institut des relations raciales, ou de certains syndicats. L'Université du Cap (UCT) a pu collecter d'importants fonds, dont une bonne partie a été anéantie dans le feu récemment ${ }^{5}$. Le dernier exemple que l'on peut retenir est celui des archives de l'African National Congress (ANC), appelées officiellement Liberation Movement Archives. Les dirigeants du mouvement en exil ont progressivement déposé leurs papiers, ainsi que d'autres documents sur le parti, à I'Université de Fort Hare (Alice). Ce centre a pu acquérir des fonds provenant d'autres mouvements de libération comme le Pan-African Congress ou le parti communiste, mais aussi auprès de personnalités ou figures de la lutte anti-apartheid.

Un dernier type d'archives s'est développé, depuis les années 1970, qui se définit comme militant. Deux exemples permettent de préciser ce qu'il faut entendre par archives militantes. Après 1994, la prison sur l'île de Robben Island a été transformée en musée. En parallèle, les documents des détenus ont trouvé place à l'Université de Western Cape (Le Cap) au sein des archives appelées Mayibuye ${ }^{6}$. Ces dernières ont acquis une fonction très importante pour la construction de la nouvelle Afrique du Sud. En outre, les South African Historical Archives (SAHA, à Johannesbourg) ont pu rassembler tout ce qui concerne les luttes en Afrique du Sud et au Zimbabwe ${ }^{7}$. Elles en sont très vite venues à se définir comme militantes, car elles ont cherché à dépasser le rôle de conservation pour s'engager avec les acteurs de la société civile, des mouvements sociaux et des protestations afin de constituer avec eux leurs archives, en plus de jouer un rôle actif dans l'accès aux archives d'État. De cette dernière institution est né le pôle archivistique de I'Université de Wits dont il est question ici. II s'inscrit dans un moment spécifique aussi de l'histoire des archives en Afrique du Sud.

\section{Le développement historique des archives indépendantes}

Pour comprendre l'importance de ces centres, leur nombre et leur capacité mobilisatrice, il faut revenir sur I'histoire politique du pays, et plus particulièrement sur la lutte contre l'apartheid. À partir des années 1970, les différentes formes d'opposition à ce régime gagnent en puissance et en visibilité globalement dans le pays. Des moments forts comme les grèves de Durban en 1973 ou les émeutes de Soweto de 1976 signalent que quelque chose change dans le pays. Ces dynamiques se reflètent aussi dans les activités universitaires et l'attraction que ces institutions développent. Le monde académique est alors divisé en plusieurs ensembles. Pour simplifier, on peut distinguer les universités anglophones de tradition libérale, parmi lesquels UCT, Rhodes, Wits, Kwazulu-Natal University (connue à l'époque comme Université du Natal). À ces dernières s'ajoutent plus tardivement les universités de Western Cape et de Fort Hare. Un autre ensemble concerne les universités afrikaners.

Au sein du premier ensemble, les années 1970 sont marquées par le développement d'un fort activisme contre le système de l'apartheid. Dans ce cadre, par exemple, un réseau de

\footnotetext{
${ }^{4}$ URL : http://www.historicalpapers.wits.ac.za/ (consulté le 5 octobre 2021).

${ }^{5}$ Le 18 avril 2021, un feu s'est déclaré dans le bâtiment de la principale bibliothèque de l'université et a rapidement gagné plusieurs sections. Le déclenchement des dispositifs anti-incendie a accru les dommages du feu, en noyant une partie des documents.

${ }^{6}$ URL : https://mayibuyearchives.org/ (consulté le 5 octobre 2021).

7 La lutte pour l'indépendance au Zimbabwe dispose d'une forte résonance pour les militants sud-africains, aussi un ensemble de documents des deux luttes intérieures est-il réuni à SAHA. URL : https://www.saha.org.za/ (consulté le 5 octobre 2021).
} 
chercheurs en sciences sociales fonde History Workshop', s'inspirant des travaux marxistes et de l'œuvre de E.P. Thompson en histoire sociale9. Ses membres ouvrent de nouveaux champs de recherche qui les amènent à multiplier les contacts auprès des acteurs des mouvements sociaux et de la société civile, eux-mêmes en plein renouvellement par les différentes luttes qui prennent forme dans les années 1980. Ce contexte explique pourquoi un nombre grandissant de personnes ou d'organisations choisissent de déposer leurs papiers au sein des centres d'archives universitaires.

Ce choix répond au statut d'autonomie des universités dont elles bénéficient depuis leur création, qui les fait apparaître comme des endroits croisant expertise et sécurité. Leurs personnels sont à même de conserver, classer, préserver les dépôts de chacun. Bien sûr, cela ne signifie pas que ces fonds deviennent accessibles à tous, surtout dans le contexte de l'apartheid. Mais pour les personnes participant à la lutte, c'est une manière de s'assurer d'une conservation relativement sûre de leurs archives. Naturellement, en retour, cela crée une responsabilité importante pour l'institution qui se voit chargée d'une tâche que les autres structures ne peuvent réaliser : I'archivage et la conservation. II faut dire qu'à l'heure de la lutte, beaucoup ne pensent tout simplement pas au devenir de leurs papiers ou craignent une confiscation.

Dans l'Afrique du Sud de la fin du régime d'apartheid, la situation des archives semble florissante. La multiplicité des centres d'archives mentionnée souligne cette richesse. II faut toutefois pondérer cette affirmation en se souvenant de la grande diversité des mouvements et des organisations qui parsèment le pays. La force de l'Afrique du Sud à ce moment tient surtout à l'attraction pour ses projets. La Truth and Reconciliation Commission donne à voir une forme originale de transition faisant de la mémoire, de la mise en avant du passé, un élément essentiel. Ceci ne signifie pas pour autant que cette institution épuise les ressources historiques de l'apartheid, bien au contraire. Plus encore, forum, séminaires, débats surgissent dans le pays, donnant naissance tout à la fois à une industrie de l'héritage ${ }^{10}$ et à une intense réflexion sur les archives, leur place dans la nouvelle société démocratique et sur l'importance de l'histoire. L'intérêt se porte encore sur l'histoire orale, sur la mémoire, ou sur la manière de faire émerger des pans entiers de l'histoire délaissée.

Cet engouement n'est nullement que local, au contraire. Ateliers de travail et conférences favorisent la venue de chercheurs, d'activistes, de membres de sociétés civiles du monde entier, voulant partager les réflexions d'une société en transformation. En parallèle, les réformes sudafricaines sont largement aidées par différentes organisations philanthropiques. Dans le cas des archives, il s'agit principalement de l'Atlantic Philanthropies, une fondation mise en place par l'irlando-américain Chuck Feeney. Les investissements se révèlent colossaux ${ }^{11}$. Alors que la

\footnotetext{
8 URL : https://www.wits.ac.za/history-workshop/ (consulté le 5 octobre 2021).

${ }^{9}$ Voir en premier lieu la dernière réédition de Thompson E. P. (2013), La formation de la classe ouvrière anglaise, Paris, Seuil. Sur le développement du Wits History Workshop, voir Bonner Philip (1994), « New nation, new history: the History workshop in South Africa, 1977-1994 », The Journal of American History, 81(3), pp. 977-985.

10 Cette dernière comprend toute une série de lieux qui sont commémorés comme symbole de l'oppression datant de l'apartheid, avec les mémoriaux de Sharpeville (en mémoire du massacre de 1960), de Sophiatown (en souvenir de l'éviction des populations coloured en 1956), etc. La multiplication de ces endroits qui redéfinissent la géographie du souvenir, prend place au sein une réflexion sur ce qu'est le patrimoine et comment le décolonialiser, pour créer une mémoire inclusive à l'exemple du nouvel État post-1994. Ce champ de recherche donne naissance aux heritage studies.

11 À titre indicatif, SAHA a pu recevoir, en 2013, un soutien financier de 434000 dollars, pour 26 mois d'exercice de cette fondation.
} 
plupart des centres d'archives indépendants fonctionnent à l'image d'ONG, avec le plus souvent un salarié et de nombreux volontaires, ces investissements autorisent le fleurissement de nombreuses initiatives. Dans le même temps, la période de transition s'accompagne de l'émergence de nouveaux mouvements et de nouvelles exigences démocratiques. Dans ce contexte, SAHA se pose non pas seulement comme un collecteur et conservateur de documents, mais comme un accompagnateur des mouvements en cours, les aidant à structurer leurs archives.

Cet enthousiasme a connu une progressive érosion à la fin des années 2000 et au début des années 2010. Aucun événement ne permet de dater le début de cette crise protéiforme que rencontre I'Afrique du Sud. Les organisations philanthropiques se sont désengagées depuis six ans, l'intérêt politique ou public pour les archives s'estompe d'autant. La prolifération des sites mémoriaux ne s'est pas accompagnée d'un engouement durable. De même, les autorités ont investi dans des lieux de prestige, ou dans des bâtiments, sans pour autant s'assurer de la maintenance ni de la pérennité de la formation des personnels et des structures de conservation. Aussi les archives d'État sont-elles en crise. Or, même si l'Afrique du Sud peut paraître comme un pays disposant d'un arsenal archivistique important, un autre constat s'impose : à l'heure de la crise - de l'État, des structures publiques - et de la profusion des initiatives citoyennes, un besoin d'archives se révèle de plus en plus urgent. Dans ce contexte, l'entreprise de hub archivistique à Wits tente de proposer une réponse originale.

\section{De l'activisme archivistique au hub}

En 2020, trois institutions ont décidé de créer une entité à même de gérer et développer en commun un pôle archivistique à l'Université de Wits : Wits History Workshop, Historical Papers et SAHA. Pour comprendre ce rapprochement et les conséquences prévisibles d'une telle création, au-delà de la crise actuelle, il faut revenir sur la distinction entre archives indépendantes et militantes, mais aussi sur la trajectoire plus spécifique de SAHA. La fondation de ce centre en 1988 tient en premier lieu aux entreprises et mouvements de libération qui mettent un terme au régime de l'apartheid, augmentés de la documentation venant du Zimbabwe. Une fois le régime d'apartheid légalement aboli en 1994, SAHA en vient à se définir comme «activiste » en plus d'être un centre indépendant. La différence tient à un certain nombre d'actions engagées.

Un premier registre d'actions relève de la promotion de l'accès aux archives. En vertu de la loi Public access information act (PAIA) ${ }^{12}$, les fonds d'État doivent être consultables par les citoyens. S'ils ne sont pas rendus accessibles, conformément à la loi, l'État peut être attaqué. SAHA porte régulièrement en justice des cas d'obstruction pour favoriser une meilleure connaissance des documents, surtout sur la période de l'apartheid. Cette activité militante s'accompagne d'une réflexion sur l'articulation entre citoyenneté et archives. À l'origine du hub de l'Université de Wits, l'organisation d'un colloque sur la place des archives, duquel est né un

\footnotetext{
12 Loi permettant l'accès à l'information pour les citoyens, qu'elle soit conservée par l'État ou des organismes privées, PAIA vise à assurer une meilleure connaissance.
} 
guide populaire sur les archives ${ }^{13}$ a marqué un jalon important. Ce guide vise à donner des outils, autant aux acteurs de la société civile qu'aux militants ou chercheurs, afin de comprendre le paysage archivistique sud-africain et les dilemmes auxquels il expose.

Cette dimension militante ne se limite pas à l'accès ou la connaissance de l'archive. Elle porte aussi sur l'accompagnement des mouvements en cours, pour les aider à constituer des archives, à les conserver et à documenter leur action. Une telle démarche ne peut se faire sans un contact régulier avec les acteurs et une pleine intégration dans leur propre dispositif de lutte. C'est ainsi que SAHA a pu constituer des fonds documentaires autour de mouvements comme Anti-Privatization Forum ${ }^{14}$. Naturellement, cette position éthique et politique impose une nette démarcation avec la plupart des bailleurs - l'État ou les compagnies privées - pour conserver une pleine latitude d'action. II existe en outre une part d'aléatoire: SAHA a par exemple tenté d'approcher les membres de Feesmustfal/ ${ }^{5}$ pour les aider à structurer leur documentation, mais aucun résultat probant n'a été obtenu pour le moment.

Au croisement du militantisme et des mutations institutionnelles est née l'idée d'un hub archivistique. Dans les années 2000, SAHA est en collaboration avec Wits University. Toute une série de facteurs tenant, entre autres, à la croissance des activités et des fonds conservés, ont poussé la direction à choisir d'autres sites que ceux de l'université. Cependant, quelque chose de nouveau a progressivement émergé ces dernières années, en lien avec une reconfiguration des aides publiques et le retrait de bailleurs comme I'Atlantic Philanthropies. La question de la place de SAHA au sein du dispositif de Wits University s'est posée. Cette université, comme espace autonome, garant de certaines pratiques et historiquement institution hébergeant SAHA dans les années 1990, s'est imposée comme l'organisation à même de "réintégrer » SAHA. Immédiatement, le vice-chancelier de l'époque, Adam Habib, a reçu avec intérêt cette nouvelle demande. L'engagement du vice-chancelier atteste de ce que représente un dépôt indépendant pour une université : plus qu'un faire-valoir, il s'agit bien d'une mission essentielle pour ces lieux de savoir.

Depuis que cette proposition a été faite, la décision a été prise de former un comité pour, non pas procéder à un changement de statut administratif, mais bien réfléchir à ce que veut dire le fait d'installer des archives au sein d'une université en Afrique du Sud post-apartheid. Cette inflexion souligne les enjeux du moment, relativement inédits. D'un côté, une série de crises modifient les capacités des centres indépendants. Le récent incendie de UCT a malheureusement démontré les dangers encourus par ces centres ne disposant pas de toutes les attentions nécessaires. De l'autre, des défis nouveaux se posent par l'essor de nouveaux supports documentaires. À l'origine de ce hub, interviennent les nouveaux outils, médias sociaux et leurs productions. Que faire des milliers de messages WhatsApp, Facebook et autres? Lors d'un mouvement comme Feesmustfall, tous ont pu témoigner de l'importance

\footnotetext{
13 Le guide est accessible en ligne. URL : https://issuu.com/hworkshop49/docs/people_s_guide_to_archives_ok (consulté le 4 octobre 2021). Pour une présentation, voir Lissoni Ariana et Rey Matthieu (2021), "Un guide pour les archives sud-africaines », 20\&21e siècle. Revue d'histoire, 149, pp. 137-141.

14 Ce mouvement réunit en 2000 un ensemble de militants, venant entre autres des townships de la province du Gauteng dans laquelle se situent Johannesburg et Pretoria. II dénonce le plan urbain proposé par la municipalité de Johannesburg, intitulé iGoli 2002, privatisant de nombreux services. II poursuit son activité pendant une décennie.

15 En 2015, les campus sud-africains sont secoués par un mouvement de contestation très important qui dénonce la hausse des frais de scolarité. Ces protestations sont désignées Feesmustfall.
} 
des supports moins classiques de mobilisation. Pourtant, aucune réflexion n'a vraiment été entamée dans les mouvements actuels ou passés sur le devenir de cette documentation. S'il est normal qu'un militant ne réfléchisse pas d'emblée, en organisant une marche, à ce que ses messages deviennent, c'est toutefois le rôle de l'historien, de l'archiviste et du citoyen. Plus encore, le fait que les archives d'État ne bénéficient plus du soutien précédent et ne peuvent donc pas proposer de réelles alternatives aux centres indépendants pour le dépôt documentaire, impose aux universités une responsabilité supplémentaire au regard de la société. Elles deviennent, plus que jamais, un lieu de savoir repensé.

Dans ce cadre, le projet de hub s'inspire aussi de ce que d'autres groupes ont tenté de faire. L'expérience du Printemps arabe a été décisive ces dernières années pour montrer l'urgence de repenser les archives. Si les projets d'archivage des mouvements arabes n'ont pas tous abouti, ils ont montré une conscience citoyenne et militante du besoin de créer de l'archive au cours de l'action ${ }^{16}$. Cette démarche participait de la rupture avec un ordre passé autoritaire. Depuis le milieu des années 2010, l'History Workshop a tenté de prendre contact avec ces groupes pour enrichir l'expérience sud-africaine. Ceci a donné lieu à un partenariat avec l'American University of Cairo, en parallèle au programme Mellon sur la Public History'7. De telles expériences n'ont pu que renforcer le projet du hub archivistique.

Ce dernier veut croiser expérience militante de l'archive et pratiques universitaires. II vise donc à créer des synergies entre les trois centres: History Workshop qui dispose de quantité d'entretiens oraux couvrant I'histoire de plusieurs dizaines de banlieues et de quartiers en Afrique du Sud; Historical Papers qui demeurent l'un des premiers centres de conservation couvrant aussi bien I'histoire du XIXe siècle que les derniers développements sud-africains ${ }^{18}$; et SAHA. Au-delà d'un simple rapprochement des expériences et des fonds documentaires, les discussions portent aussi sur le futur : comment former des étudiants aux archives ? Comment sensibiliser les nouvelles générations aux défis actuels ? Comment renforcer les dialogues avec des partenaires internationaux ? L'idée est bien de mettre en place, à terme, des cursus plus focalisés sur les archives, afin de s'assurer que la génération actuelle de directrices et directeurs trouve rapidement une relève. En effet, à l'heure de la recherche d'un potentiel employé pour le hub, aucun nom en Afrique du Sud ne s'est imposé, soulignant le manque criant de formations et d'anticipations autour de ces outils, pourtant essentiels à la construction du futur en Afrique du Sud.

La place de ce hub archivistique ne peut se comprendre sans un regard sur l'Afrique du Sud depuis la fin de l'apartheid. La situation de l'histoire et des archives y semble privilégiée au regard de ses voisins et de nombreux États. Elle dénote les espoirs et les soutiens qu'a suscité ce pays lors de la transition des années 1990. Pourtant, de façon lancinante, quelque chose s'est brisé au cours de la dernière décennie. Réforme étatique, désengagement de partenaires étrangers, moindre intérêt pour les expériences sud-africaines - devenues peutêtre plus banales sur la scène internationale -, crise multiple du politique, tout cela concourt à

\footnotetext{
${ }^{16}$ Voir, par exemple, dans le cas de l'Égypte, URL : http://tahrirarchives.com/ (consulté le 4 octobre 2021); dans le cas de la Syrie, URL : https://creativememory.org/en/archives/ (consulté le 4 octobre 2021).

17 URL : https://www.wits.ac.za/history-workshop/the-everyday-and-public-history/ (consulté le 4 octobre 2021).

18 Historical Papers conserve un ensemble de fonds, soit d'organisations (Anglican Church) soit d'individus.
} 
menacer cette tradition de l'archive au service d'une citoyenneté repensée. Pour tenter d'apporter une réponse à ces défis, qui ne peut suffire à elle seule mais qui atteste des volontés universitaires et militantes, un pôle archivistique tente d'opérer actuellement un rassemblement majeur de fonds d'archives au service d'un nouveau regard sur les centres indépendants de documentation et les pratiques qu'ils doivent générer.

Noor Nieftegodien

History Workshop, Université du Witwatersrand (Afrique du Sud) Matthieu Rey IFPO Beyrouth / History Workshop, Université du Witwatersrand (Afrique du Sud)

\section{Bibliographie}

BONNER Philip (1994), « New nation, new history: the History workshop in South Africa, 1977-1994 », The Journal of American History, 81(3), pp. 977-985.

LISSONI Ariana et ReY Matthieu (2021), "Un guide pour les archives sud-africaines », 20\&21e siècle. Revue d'histoire, 149, pp. 137-141.

ThOMPSOn E. P. (2013), La formation de la classe ouvrière anglaise, Paris, Seuil. 